
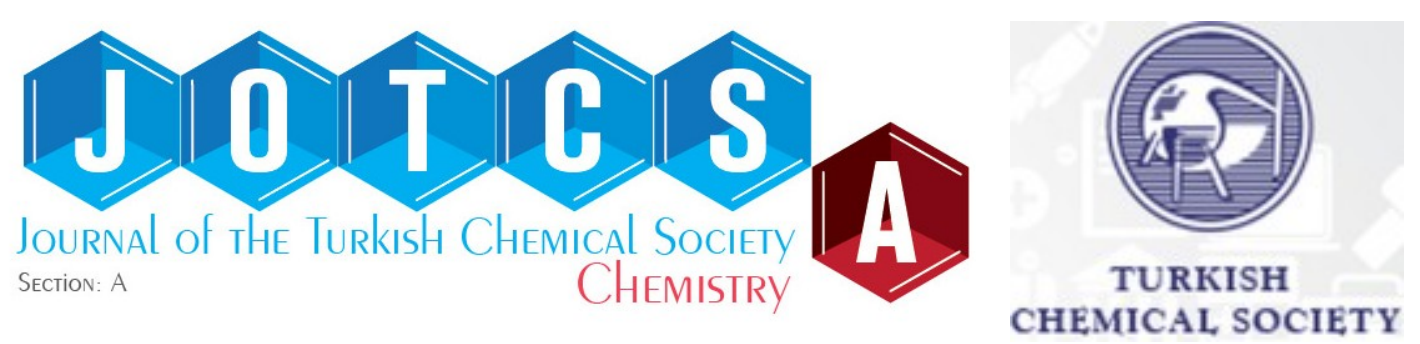

\title{
Computational Screening of Covalent Organic Frameworks for Hydrogen Storage
}

\author{
Ezgi Gulcay $^{1}$ and İlknur Erucar ${ }^{2} * \square$ \\ ${ }^{1}$ Ozyegin University, Faculty of Engineering, Department of Mechanical Engineering, 34794, Istanbul, \\ Turkey. \\ ${ }^{2}$ Ozyegin University, Faculty of Engineering, Department of Natural and Mathematical Sciences, 34794, \\ Istanbul, Turkey.
}

\begin{abstract}
Covalent Organic Frameworks (COFs) have been considered as promising materials for gas storage applications due to their highly porous structures and tunable characteristics. In this work, high-throughput molecular simulations were performed to screen the recent Computation-Ready Experimental COF Database (CoRE-COF) for $\mathrm{H}_{2}$ storage as a first time in the literature. Predictions for $\mathrm{H}_{2}$ uptakes were first compared with the experimental data of several COFs. Motivated from the good agreement between simulations and experiments, we performed Grand Canonical Monte Carlo (GCMC) simulations to compute volumetric $\mathrm{H}_{2}$ uptakes of 296 COFs at various temperatures and pressures and identified the best candidates which exhibit superior performance for $\mathrm{H}_{2}$ storage. COFs outperformed several well-known MOFs such as HKUST-1, NU-125, NU-1000 series, NOTT-112 and UiO-67 at 100 bar/77 K adsorption and 5 bar/160 K desorption conditions. We also examined the effect of FeynmanHibbs correction on simulated $\mathrm{H}_{2}$ isotherms and $\mathrm{H}_{2}$ working capacities of COFs to consider quantum effects at low temperatures. Results showed that the Feynman-Hibbs corrections do not affect the ranking of materials based on $\mathrm{H}_{2}$ working capacities, but slightly affect the predictions of $\mathrm{H}_{2}$ adsorption isotherms. We finally examined the structure-performance relations and showed that density and porosity are highly correlated with the volumetric $\mathrm{H}_{2}$ working capacities of COFs. Results of this study will be highly useful in guiding future research and focusing experimental efforts on the best COF adsorbents identified in this study.
\end{abstract}

Keywords: Covalent organic frameworks, Molecular simulations, Hydrogen storage, Working capacity, Adsorption..

Submitted: May 15, 2019. Accepted: October 27, 2019.

Cite this: Gulcay E, Erucar, İ. Computational screening of covalent organic frameworks for hydrogen storage. JOTCSA. 2020; 7(1): 65-76.

DOI: https://doi.org/10.18596/jotcsa.565460.

Corresponding Author. E-mail: ilknur.erucar@ozyegin.edu.tr, Tel: +90 (216) 564-9297.

\section{INTRODUCTION}

Developing energy-efficient and safe hydrogen $\left(\mathrm{H}_{2}\right)$ storage systems has gained importance in transportation sector due to non-toxic and environmentally friendly products of $\mathrm{H}_{2}$ compared to those of fossil fuels. Additionally, $\mathrm{H}_{2}$ has almost triple energy density that of gasoline per mass unit. Many companies including Honda, Toyota, Hyundai, and General Motors have been recently producing hydrogen-powered vehicles (1). Storing $\mathrm{H}_{2}$ in vehicles is possible at room temperature and very high pressure ( $~ 700$ bar). However, high pressure operating conditions bring safety issues to the attention of the manufacturers for the critical equipment design. 
As alternative to the high-pressure $\mathrm{H}_{2}$ storage systems, liquefaction at low temperature (77 K) has been also investigated for on-board $\mathrm{H}_{2}$ storage. Recently, cryo-adsorption processes in which $\mathrm{H}_{2}$ molecules are stored at $77 \mathrm{~K}$ and 100 bar and desorbed at $160 \mathrm{~K}$ and 5 bar using a porous material have been considered for automotive industry (2). $\mathrm{H}_{2}$ molecules can be adsorbed at moderate temperatures and pressures within a porous adsorbent material due to the van der Waals interactions. Herein, the careful choice of an adsorbent material is important. A promising adsorbent should have high working capacity. Working capacity is defined as the gas amount that can be delivered when the storage pressure is decreased to a predetermined desorption pressure (3). To have a high gas working capacity, adsorbents should have the maximum gas uptake at an adsorption pressure and the minimum gas uptake at a desorption pressure.

Many different adsorbent materials including activated carbons, (4) zeolites, (5) metal organic frameworks (MOFs) (6) and covalent organic frameworks (COFs) $(7,8)$ have been tested for efficient $\mathrm{H}_{2}$ store systems. To evaluate $\mathrm{H}_{2}$ storage capacities of these materials, ARPA-E (Advanced Research Projects Agency-Energy) target set by the U.S. Department of Energy (DOE) has been commonly used. The DOE target for on board $\mathrm{H}_{2}$ storage systems was set to $4.5 \mathrm{wt} \% ; 30 \mathrm{~g} / \mathrm{L}$ for 2020 and $5.5 \mathrm{wt} \% ; 40 \mathrm{~g} / \mathrm{L}$ for 2025 and $6.5 \mathrm{wt}$ $\% ; 50 \mathrm{~g} / \mathrm{L}$ as ultimate target (9). Among these adsorbent materials, MOFs constructed from metal ions and organic linkers via coordination bonds are potential candidates for $\mathrm{H}_{2}$ storage due to their large surface areas and high pore volumes (10). For example, Gómez-Gualdrón et al. (2) investigated the isoreticular series of zirconium MOFs including NU-1101, NU-1102 and NU-1103 (NU: Northwestern University) and found that $\mathrm{NU}-1101$ gives the highest measured volumetric $\mathrm{H}_{2}$ uptake as $46.6 \mathrm{~g} / \mathrm{L}$ (9.1 wt \%), whereas NU-1103 gives the highest gravimetric $\mathrm{H}_{2}$ uptake as with $12.6 \mathrm{wt} \%(43.2 \mathrm{~g} / \mathrm{L})$ at 77 $\mathrm{K} / 100$ bar adsorption and $160 \mathrm{~K} / 5$ bar desorption conditions based on the tank design criteria proposed by HSECoE, Hydrogen Storage Engineering Center of Excellence (11). Langmi et al. (12) reviewed $\mathrm{H}_{2}$ uptakes in MOFs at both 77 $\mathrm{K}$ and $298 \mathrm{~K}$ and showed that MOFs provide total volumetric $\mathrm{H}_{2}$ uptake in the range of $40-60 \mathrm{~g} / \mathrm{L}$ (6-15 wt\%) at $77 \mathrm{~K}$ whereas it decreases to less than $15 \mathrm{~g} / \mathrm{L}(0.5-1 \mathrm{wt} \%)$ at $298 \mathrm{~K}$. COFs as a sub class of MOFs have been also considered as promising adsorbents for $\mathrm{H}_{2}$ storage. COFs are consisted of light elements including B (boron), C (carbon), $\mathrm{N}$ (nitrogen) and $\mathrm{O}$ (oxygen) and organic linkers which are covalently bonded. COFs exhibit large surface areas (711-1590 $\left.\mathrm{m}^{2} / \mathrm{g}\right)$, high porosities (0.25-0.94) and large pore sizes $(7.0 \times 27.0 \AA)(13)$. Furukawa et al. (14) examined the $\mathrm{H}_{2}$ storage performances of seven COFs including COF-1, COF-5, COF-6, COF-8, COF-10, COF-102 and COF-103 at $77 \mathrm{~K}$. They found that saturated $\mathrm{H}_{2}$ uptakes of these COFs are in the range of $1.5 \mathrm{wt} \%$ to $7.2 \mathrm{wt} \%$. Ding and Wang (15) summarized the $\mathrm{H}_{2}$ uptakes of thirteen COFs and reported that COFs with larger surface areas exhibit higher $\mathrm{H}_{2}$ uptake capacities. All these studies showed that developing novel adsorbents which have high storage and working capacity for $\mathrm{H}_{2}$ at ambient conditions has been still a critical issue for practical applications.

Both MOFs and COFs can be synthesized using the reticular design concept which provides numerous materials with different chemical functionalities. Evaluating the performance of a large number of materials for $\mathrm{H}_{2}$ storage using experimental methods is challenging due to time, cost, and equipment concerns. Therefore, molecular simulations play a very useful role for providing reliable gas adsorption data in a reasonable time. Many computational studies on $\mathrm{H}_{2}$ uptake in MOFs were performed in the literature (16). Bucior et al. (1) performed a large-scale molecular simulation study to identify promising candidates for $\mathrm{H}_{2}$ storage. Among 54,776 MOFs, 25 materials were reported to exceed the DOE 2025 volumetric system target $(40 \mathrm{~g} / \mathrm{L})$. In a different study, Ahmed et al. (17) screened real and hypothetical MOFs (totally 500,000 MOFs) for $\mathrm{H}_{2}$ adsorption up to 100 bar at $77 \mathrm{~K}$. Among these halves million MOFs, NU100 (also known as PCN-610, PCN for porous coordination network) surpassed the DOE's 2020 system level target $\left(30 \mathrm{~g} / \mathrm{L}\right.$ ) and gave $\mathrm{H}_{2}$ uptake almost $35 \mathrm{~g} / \mathrm{L}$. They also investigated the relation between structural properties of MOFs and their performances and reported that porosity has positively correlated with the volumetric working capacity of MOFs. Six different COFs (COF-1, 5, $102,103,105$ and 108) have also been tested for $\mathrm{H}_{2}$ storage by Han et al. (7) due to their large surface areas (up to $\sim 4000 \mathrm{~m}^{2} / \mathrm{g}$ ) and low crystal densities $\left(\sim 0.2 \mathrm{~g} / \mathrm{cm}^{3}\right)$. Among these COFs, COF108 gave the highest $\mathrm{H}_{2}$ gravimetric uptake ( 19 wt $\%)$ and COF-102 gave the maximum volumetric $\mathrm{H}_{2}$ uptake ( $\left.40 \mathrm{~g} / \mathrm{L}\right)$ at $77 \mathrm{~K}$ and 100 bar. In a recent study, Cao et al. (18) performed simulations of four different three-dimensional (3D) COFs (COF-102, 103, 105 and 108) and their lithium (Li)-doped counterparts for $\mathrm{H}_{2}$ storage at $298 \mathrm{~K}$ up to 100 bar. Results showed that $\mathrm{H}_{2}$ gravimetric uptake capacities of Li-doped COFs (COF-105 and COF-108) nearly doubled and both reached $\sim 7$ wt\% at ambient temperature due to the favorable interactions between $\mathrm{H}_{2}$ and $\mathrm{Li}$ atom. Assfour et al. (19) performed molecular simulations of eleven COFs for $\mathrm{H}_{2}$ storage at $77 \mathrm{~K}$ and $298 \mathrm{~K}$ up to 100 bar. 
Similar to study of Han et. al. (7), they found that COF-108 has the highest gravimetric $\mathrm{H}_{2}$ uptake as 21 wt $\%$ and 4.17 wt $\%$ at $77 \mathrm{~K}$ and 100 bar and at $298 \mathrm{~K}$ and 100 bar, respectively. These results suggest that COFs can be promising materials for $\mathrm{H}_{2}$ storage. Currently, 309 COFs were deposited in the ComputationReady Experimental COF Database (CORE-COF) (20) and to the best of our knowledge, there is no study in the literature which evaluates $\mathrm{H}_{2}$ storage performances of COFs by using highthroughput molecular simulation techniques. Therefore, computational studies will be highly useful to identify the potential COF candidates for $\mathrm{H}_{2}$ capture.

In this work, we screened the recent CoRE-COF database (20) for $\mathrm{H}_{2}$ storage as a first time in the literature. We first compared our predictions with the available experimental data in the literature for $\mathrm{H}_{2}$ uptake. We examined the effect of Feynman-Hibbs corrections on simulated $\mathrm{H}_{2}$ isotherms in COFs. In many computational studies on $\mathrm{H}_{2}$ storage, $\mathrm{H}_{2}$ molecules are treated as classical molecules. However, at sufficiently low temperatures, quantum effects should be considered in simulations due to the low mass of $\mathrm{H}_{2}$. We then performed Grand Canonical Monte Carlo (GCMC) simulations to compute volumetric $\mathrm{H}_{2}$ uptakes of 296 COFs at three different operating conditions: (i) at $100 \mathrm{bar} / 77 \mathrm{~K} \rightarrow 2 \mathrm{bar} /$ $77 \mathrm{~K}$, (ii) at $100 \mathrm{bar} / 77 \mathrm{~K} \rightarrow 5 \mathrm{bar} / 77 \mathrm{~K}$, and (iii) at $100 \mathrm{bar} / 77 \mathrm{~K} \rightarrow 5 \mathrm{bar} / 160 \mathrm{~K}$. Reporting volumetric $\mathrm{H}_{2}$ adsorption is crucial because it directly relates to the required volume of an onboard tank. We also investigated the effect of the Feynman-Hibbs correction on simulated $\mathrm{H}_{2}$ working capacities of COFs and the ranking of the best materials. The best performing materials were then compared with the top performing MOFs which were previously identified in the literature. We finally examined the relations between structural properties of COFs such as pore sizes, densities, porosities and their $\mathrm{H}_{2}$ working capacities to provide structureperformance relationships.

\section{MATERIALS AND METHODS}

\section{Computational details}

To validate our computational methodology, we first compared our predictions with the available experimental data of Furukawa et al. (14) for single-component $\mathrm{H}_{2}$ adsorption. Adsorption isotherms of $\mathrm{H}_{2}$ in four different COFs (COF-5, COF-6, COF-8 and COF-10) were computed at 77 $\mathrm{K}$ up to 80 bar to be consistent with the study of Furukawa et al. (14). We also computed saturated $\mathrm{H}_{2}$ uptakes in several COFs reported in the literature by $\mathrm{Li}$ et al. (21) (ACOF-1), Stegbauer et al. (22) (ATFG-COF), Furukawa et al. (14) (COF-1, COF-5, COF-6, COF-8, COF-10, COF-102, and COF-103), Li et al. (23) (COFJLU2), Ge et al. (24) (COF-TpAzo), Neti et al. (25) (CoPc-PorDBA), Kaleeswaran et al. (26) (iPrTAPB-TFP, iPrTAPB-TFPB, TAPB-TFP, and TAPB-TFPB), Kang et al. (27) (NUS-3), Bhunia et al. (28) (PCTF-n, $n=1-2)$, and Kahveci et al. (29) (TD-COF-5). All the crystal structures of COFs were taken from CoRE-COF database.(20) To compare our simulation results and experimental values, the absolute adsorbed gas amount ( $n_{a b s}$ ), which could not be directly measured, was converted to the excess adsorption $\left(n_{e x}\right)$.(3) The excess adsorption can be explained by the difference between the amount of the absolute adsorption, and also bulk gas in the adsorbed region and calculated from Equation 1:

$$
n_{e x}=n_{\mathrm{abs}}-V_{p} \cdot \rho_{\text {bulk }}(P, T) \text { (Eq. 1) }
$$

Herein, $\mathrm{n}_{\mathrm{ex}}$ is the excess adsorption, $\mathrm{n}_{\mathrm{abs}}$ is the absolute adsorbed gas amount, $V_{p}$ is the pore volume $\left(\mathrm{cm}^{3} / \mathrm{g}\right)$ and $\rho$ bulk represents the density of the gas in the bulk phase calculated with the Peng-Robinson equation of state at temperature $(T)$ and pressure $(P)$, respectively.

The crystal structures of 309 COFs were taken from the solvent-free CoRE-COF database (20). The largest cavity diameter (LCD), pore-limiting diameter (PLD), surface area (SA), density, pore volume (PV) and porosity $(\phi)$ were calculated by Zeo++ software (30). SA calculations were performed using nitrogen kinetic diameter as $1.86 \AA$ and the trial number was set to 2000 . For PV calculations, zero probe size was used, and the number of trials was set to 50,000. Among 309 COFs, 13 COFs have almost zero accessible SAs and these frameworks were excluded from further $\mathrm{H}_{2}$ adsorption analysis.

Grand Canonical Monte Carlo (GCMC) simulations were performed to compute single-component $\mathrm{H}_{2}$ adsorption in 296 COFs at various temperatures ( $77 \mathrm{~K}$ and $160 \mathrm{~K}$ ) and pressures ( 2 bar, 5 bar and 100 bar) as implemented in RASPA software (31). Three different types of moves including translation, reinsertion, and swap of the molecule were considered in GCMC simulations. Rotation move was also applied in GCMC simulations for the three-site model of $\mathrm{H}_{2}$. The Lorentz-Berthelot mixing rules were employed to calculate pair wise interactions. The Peng-Robinson equation of state was used to convert the pressure to the corresponding fugacity. Simulations were carried out for a total of $10^{4}$ cycles with 3000 cycles for the equilibration. Lennard-Jones (LJ) $12-6$ and Coulombic potentials were used to model repulsion-dispersion forces, and electrostatic interactions, respectively using Equation 2 as follows: 


$$
U_{i j(r)}=4 \epsilon_{i j}\left(\left(\frac{\sigma_{i j}}{r}\right)^{12}-\left(\frac{\sigma_{i j}}{r}\right)^{6}\right)+\frac{q_{i} q_{j}}{4 \pi \epsilon_{0} r} \text { (Eq. 2) }
$$

where $U_{i j}$ represents the potential energy between atoms $i$ and $j, r$ is the separation distance from the center of one particle to the center of the other particle, $\varepsilon_{\mathrm{ij}}$ the well depth and $\sigma_{i j}$ is the molecular length scale based on the particle diameter. In Equation $2 \varepsilon_{0}$, qi and $q j$ show the electric constant, partial atomic charges of $i$ and $j$, respectively. The cut-off radius for truncation was set to $12.8 \AA$. The simulation cell lengths were increased to at least $25.6 \AA$ along each dimension. To compute electrostatic interactions between gas molecules and the frameworks' atoms, the partial atomic charges of materials were estimated using the charge equilibration method as implemented in RASPA (31). Ewald's summation (32) was used for the long-range electrostatic calculations. $\mathrm{H}_{2}$ molecules were modeled using two different models including a single-site model (33), and three-site linear molecule with two sites located at two atoms and the third one located at its center of mass (COM) (34). The interaction parameters of $\mathrm{H}_{2}$ used in molecular simulations were given in Table 1.

Table 1: The interaction parameters and partial charges used for $\mathrm{H}_{2}$ molecules.

\begin{tabular}{lllll}
\hline Molecule & Site & $\boldsymbol{\varepsilon} / \mathbf{k B}(\mathbf{K})$ & $\boldsymbol{\sigma}(\boldsymbol{\AA})$ & $\mathbf{q}(\mathbf{e})$ \\
\hline $\mathrm{H}_{2}$ & Center of Mass (COM) & 36.700 & 2.958 & -0.936 \\
& $\mathrm{H}$ & 0.000 & 0.000 & 0.468 \\
& Single-site & 34.200 & 2.960 & 0.000 \\
\hline
\end{tabular}

The Feynman-Hibbs correction (35) given in Equation 3 was applied to include quantum effects at $77 \mathrm{~K}$.

$$
U_{F H(r)}=U_{I J(r)}+U_{\operatorname{coul}(r)}+\frac{\hbar^{2}}{24 \mu K T} \nabla^{2} U_{I J(r)} \text { (Eq. 3) }
$$

In Equation 3, $U_{F H(r)}$ the potential energy calculated with the Feynman-Hibbs correction, $U_{\sqcup(r)}$ is the Lennard-Jones ( $L J) R-X$ potential and $U_{\text {coul }(r)}$ is the Coulobic potential shown is Equation (2). $\hbar$ is the Planck constant divided by $2 \pi, \mu$ is the reduced mass, $\mathrm{K}$ is the Boltzman constant and $\mathrm{T}$ is the absolute temperature.

The potential parameters of COFs were taken from the Universal Force Field (UFF) (36). This force field was chosen based on the results of previous gas uptake predictions that gave a good agreement with experiments.(37)

$\mathrm{H}_{2}$ working capacities ( $W C_{\mathrm{H}_{2}}$ ) were calculated for 296 COFs, which have SAs $>0 \mathrm{~m}^{2} / \mathrm{g}$, using the following equation:

$W C_{H_{2}}=N_{a d s}-N_{d e s}$

where, $W C_{H 2}$ is the $\mathrm{H}_{2}$ working capacity $(\mathrm{g} / \mathrm{L})$, $\mathrm{Nads}$ is the adsorbed gas amount calculated at the adsorption pressure and $\mathrm{N}_{\text {des }}$ is the adsorbed gas amount $(\mathrm{g} / \mathrm{L})$ calculated at the desorption pressure.

\section{RESULTS AND DISCUSSION}

\section{Comparisons of experiments with simula- tions:}

Figure 1 shows the comparison of our predictions with the experimental measurements of COF-5, COF-6, COF-8 and COF-10 for single-component $\mathrm{H}_{2}$ adsorption at $77 \mathrm{~K}$. Simulations were performed by considering three different scenarios: all electrostatic interactions are on, $\mathrm{H}_{2}-\mathrm{H}_{2}$ electrostatic interactions are on and all electrostatic interactions are off. For the first case (all electrostatic interactions are on), $\mathrm{H}_{2}-\mathrm{H}_{2}$ electrostatic interactions using the Darkrim and Levesque potential and $\mathrm{H}_{2}$-framework electrostatic interactions were computed during GCMC simulations and the Feynman-Hibbs correction was added into the potential energy. For the second case $\left(\mathrm{H}_{2}-\mathrm{H}_{2}\right.$ electrostatic interactions are on), only $\mathrm{H}_{2}-$ $\mathrm{H}_{2}$ electrostatic interactions were considered using the Darkrim and Levesque potential (34) and the Feynman-Hibbs correction was applied during simulations. For the last case (all electrostatic interactions are off), a simple single-site LJ potential for $\mathrm{H}_{2}$ was used and the Feynman-Hibbs correction was not applied in simulations. As shown in Figure 1, simulations performed using Feynman-Hibbs corrections agreed well with the experiments. Simulations performed using the single-site $\mathrm{H}_{2}$ model overestimated $\mathrm{H}_{2}$ uptake in all these COFs. For example, Furukawa et al. (14) measured $35.1 \mathrm{mg} \mathrm{H}_{2} / \mathrm{g} \mathrm{COF}-5$ at 80 bar and 77 $\mathrm{K}$, we predicted $\mathrm{H}_{2}$ uptake in COF-5 using the single-site model as $41.2 \mathrm{mg} / \mathrm{g}$ under the same conditions. Results obtained from simulations with the Feynman-Hibbs corrections were found to be similar. The predicted $\mathrm{H}_{2}$ uptakes in COF-5 obtained 

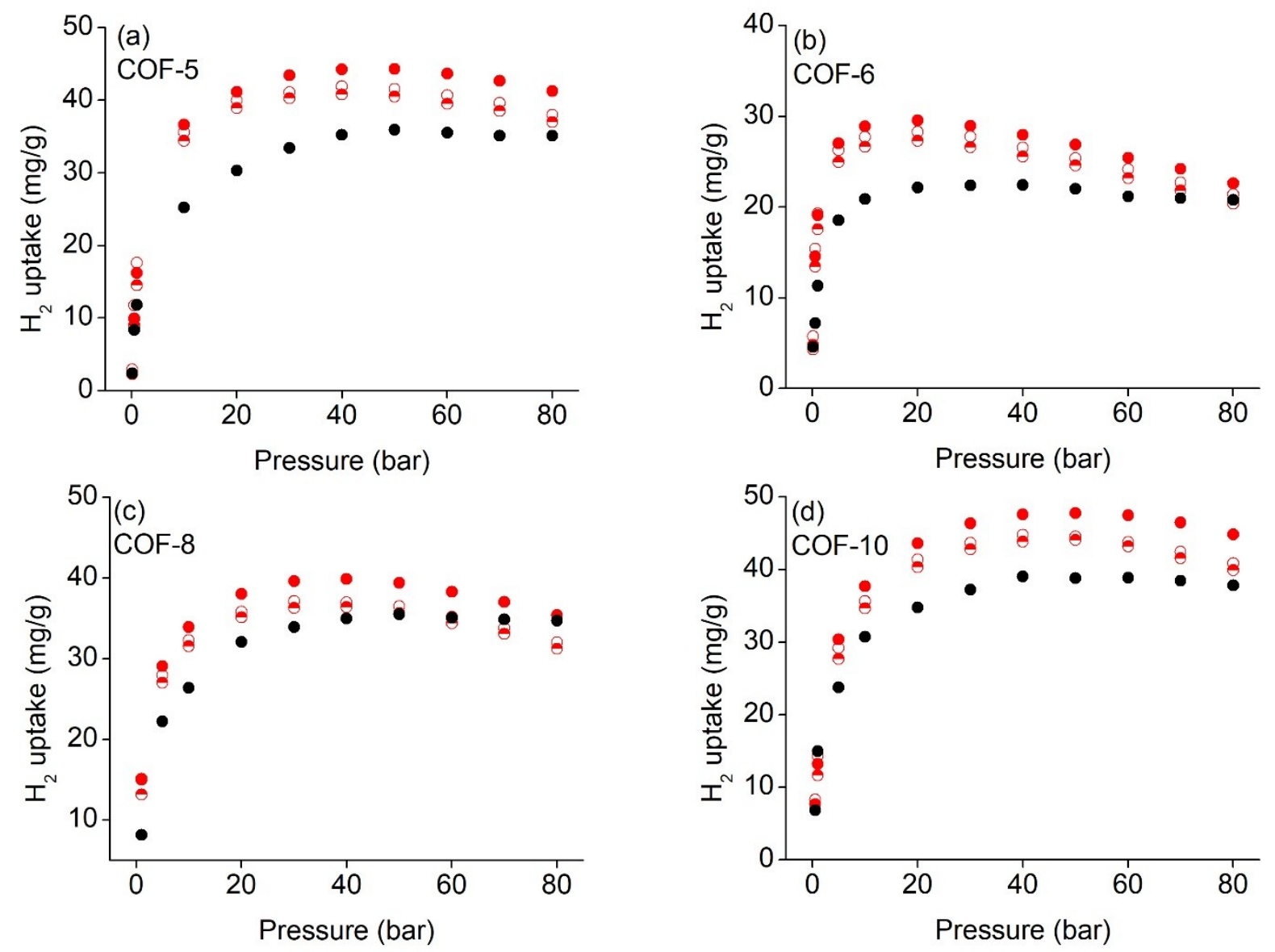

Figure 1: Comparison of our simulations with the experiments (14) for single-component $\mathrm{H}_{2}$ adsorption in (a) COF-5, (b) COF-6, (c) COF-8 and (d) COF-10 at 77K. Open, half-closed and closed red spheres represent our predictions obtained from all electrostatic interactions were on, only $\mathrm{H}_{2}-\mathrm{H}_{2}$ electrostatic interactions were on, and all electrostatic interactions were off, respectively. Black spheres represent the experimental data measured by Furukawa et al. (14)

from the simulations with the Feynman-Hibbs corrections were found as $37.0 \mathrm{mg} / \mathrm{g} \mathrm{H}_{2}-\mathrm{H}_{2}$ electrostatic interactions are on) and $38.0 \mathrm{mg} / \mathrm{g}$ (all electrostatic interactions are on) at the same conditions. Results showed that instead of assigning partial charges of COFs which requires high computation cost, only $\mathrm{H}_{2}-\mathrm{H}_{2}$ electrostatic interactions may be considered to compute $\mathrm{H}_{2}$ adsorption in COFs at cryogenic conditions in a reasonable time. Similar results were also found by Assfour et al. (19) who emphasized that coulombic interactions between $\mathrm{H}_{2}-\mathrm{H}_{2}$ molecules and COFs do not affect the $\mathrm{H}_{2}$ uptake capacities of several COFs at both $77 \mathrm{~K}$ and $298 \mathrm{~K}$.

We also compared our predictions with the experiments for the saturated $\mathrm{H}_{2}$ uptake capacities of 20 COFs in Figure 2. COFs' names together with the calculated and measured $\mathrm{H}_{2}$ uptakes at the various temperatures and pressures were given in Table 2. Among these COFs, only COF102 and COF-103 are 3D and the remaining COFs are all 2D. Simulated $\mathrm{H}_{2}$ uptakes were obtained from the GCMC simulations with the FeynmanHibbs corrections using the Darkrim and Levesque potential for $\mathrm{H}_{2}$ (only $\mathrm{H}_{2}-\mathrm{H}_{2}$ electrostatic interactions are on). The good agreement between simulations and experiments for saturated $\mathrm{H}_{2}$ uptakes in these COFs was found. The large discrepancy was observed between simulated $\mathrm{H}_{2}$ uptake and experimental measurements for only 3D COFs, COF-102 and COF-103. Simulations dramatically overestimated $\mathrm{H}_{2}$ uptake in COF-102 and COF-103 which may be attributed to the remaining solvent molecules and defects inside these materials. Overall, motivated from the good agreement between our simulations and the experimental measurements, we further computed $\mathrm{H}_{2}$ working capacities of 296 experimental COFs and discussed below. 
Table 2: Data for comparison of simulations with the experiments for the saturated $\mathrm{H}_{2}$ uptake capacities of 20 COFs.

\begin{tabular}{|c|c|c|c|c|c|}
\hline COF Name & P (bar) & $\mathbf{T}(\mathbf{K})$ & $\begin{array}{l}\text { Our data } \\
\text { (mg/g) }\end{array}$ & $\begin{array}{l}\text { Literature Data } \\
(\mathrm{mg} / \mathrm{g})\end{array}$ & Reference \\
\hline $\mathrm{ACOF}-1^{[1]}$ & 1 & 77 & 16.89 & 9.89 & $(21)$ \\
\hline ATFG-COF[2] & 30 & 308 & 2.14 & 8.12 & $(22)$ \\
\hline COF-1 & 90 & 77 & 20.24 & 14.68 & $(14)$ \\
\hline COF-5 & 90 & 77 & 35.30 & 34.19 & $(14)$ \\
\hline COF-6 & 90 & 77 & 18.97 & 20.04 & $(14)$ \\
\hline COF-8 & 80 & 77 & 31.23 & 33.85 & $(14)$ \\
\hline COF-10 & 80 & 77 & 39.91 & 37.58 & (14) \\
\hline COF-102 & 90 & 77 & 85.41 & 67.07 & $(14)$ \\
\hline COF-103 & 90 & 77 & 95.79 & 65.05 & $(14)$ \\
\hline COF-JLU2 ${ }^{[3]}$ & 1 & 77 & 12.48 & 15.86 & $(23)$ \\
\hline COF-TpAzo ${ }^{[4]}$ & 1 & 77 & 13.61 & 10.29 & $(24)$ \\
\hline CoPc-PorDBA ${ }^{[5]}$ & 1 & 77 & 41.43 & 41.24 & $(25)$ \\
\hline iPrTAPB-TFP[6] & 1 & 77 & 7.09 & 11.42 & $(26)$ \\
\hline iPrTAPB-TFPB[7] & 1 & 77 & 8.15 & 4.18 & $(26)$ \\
\hline TAPB-TFP & 1 & 77 & 14.90 & 10.82 & $(26)$ \\
\hline TAPB-TFPB & 1 & 77 & 9.63 & 6.26 & $(26)$ \\
\hline NUS-3 ${ }^{[8]}$ & 1 & 273 & 0.14 & 0.24 & $(27)$ \\
\hline PCTF- $1^{[9]}$ & 0.25 & 77 & 5.31 & 9.75 & $(28)$ \\
\hline PCTF-2 & 0.5 & 77 & 8.67 & 6.86 & $(28)$ \\
\hline TD-COF-5[10] & 1 & 77 & 27.88 & 15.89 & (29) \\
\hline
\end{tabular}

[1]ACOF: azine-based COF; [2]ATFG: 1,3,5-triformylphloroglucinol; [3]JLU: Jilin University; [4]TpAzo: triformylphloroglucinol 4,4'-azodianiline; [5]CoPc-PorDBA: cobalt-based phthalocyanine- porphyrin dehydrobenzoannulenes; [6]iPrTAPB-TFP: 1,3,5-tris(4- aminophenyl)benzene - 1,3,5-

triformylphluroglucinol; [7] TFPB: 1,3,5-tris(4'-formylphenyl)benzene; [8]NUS: : National University of Singapore; [9]PCTF: porous covalent triazine-based organic frameworks [10]TD: triptycene-derived.

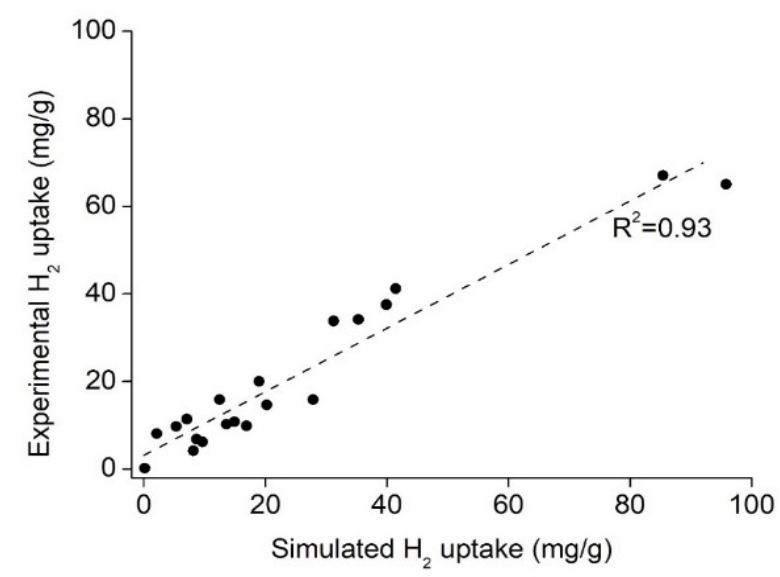

Figure 2: Comparison of experiments and our simulations for the saturated $\mathrm{H}_{2}$ uptakes in different 20 COFs.

Effect of Feynman-Hibbs correction on simulated $\mathrm{H}_{2}$ working capacities of COFs
Working capacity is a useful metric to evaluate adsorbents for gas separation applications. Classical $L$ J potential and a simple spherical $\mathrm{H}_{2}$ model are commonly used in simulations to compute $\mathrm{H}_{2}$ working capacities of adsorbents at $77 \mathrm{~K}$ in the literature. However, at low temperatures quantum effects can be important for small molecules. Therefore, the Feynman-Hibbs correction can be used to account quantum effects at low temperatures in simulations. In Figure 3, we compared $\mathrm{H}_{2}$ working capacities of 296 COFs calculated at three different operating conditions (a) from 100 bar/77 K to 2 bar/77 K, (b) from 100 bar/77 K to 5 bar/77 K, and (c) from 100 bar/77 K to 5 bar/ $160 \mathrm{~K}$ based on their potential energies. Figure 3 shows that simulation results obtained from the simulations using the Feynman-Hibbs corrections were found to be almost similar with the results obtained from the simulations using the singlesite $\mathrm{H}_{2}$ model at three different operating conditions. 
There are obvious linear relationships between the results obtained from only $L]$ potential and the results obtained from $L J$ potential with Feynman-Hibbs corrections. We then examined the ranking of COFs based on $\mathrm{H}_{2}$ working capacities calculated from only LJ potential and LJ potential with the Feynman-Hibbs corrections and estimated the Spearman's rank correlation coefficient $(\mathrm{SRCC})(-1 \leq \mathrm{SRCC} \leq 1)$. When SRCC is 1 , there is a perfect correlation between two rankings. Table 3 shows the comparison of ranking of COFs based on volumetric $\mathrm{H}_{2}$ working capacities calculated from GCMC simulations using different potentials. As shown in Table 3, the ranking of COFs is highly correlated, and the FeynmanHibbs correction and adsorbate-adsorbent electrostatic interactions do not actually affect the ranking of materials. Therefore, we can conclude that predictions using only $L J$ potential with the single-site $\mathrm{H}_{2}$ model can give quick and reliable information about the volumetric $\mathrm{H}_{2}$ working capacities of COFs.
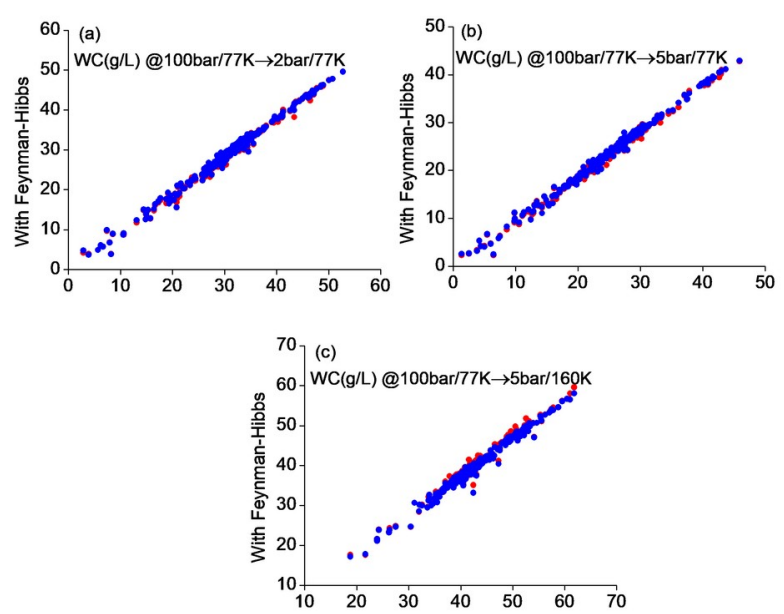

Figure 3: Comparison of $\mathrm{H}_{2}$ WCs of 296 COFs calculated with the Lennard-Jones potential only ( $x$-axis) and the Feynman-Hibbs corrections ( $y$ axis). Red (blue) points represent our predictions obtained from all electrostatic interactions were on (only $\mathrm{H}_{2}-\mathrm{H}_{2}$ electrostatic interactions were on).

Table 3: Comparison of the ranking of COFs based on their volumetric $\mathrm{H}_{2}$ working capacities.

\begin{tabular}{lcc}
\hline Adsorption/desorption conditions & $\begin{array}{c}\text { LJ vs LJ with the FH* } \\
\text { (all electrostatic interac- } \\
\text { tions) }\end{array}$ & $\begin{array}{c}\text { LJ vs LJ with the FH } \\
\text { (only H2-H2 electrostatic inter- } \\
\text { actions) }\end{array}$ \\
\hline $100 \mathrm{bar} / 77 \mathrm{~K} \rightarrow 2 \mathrm{bar} / 77 \mathrm{~K}$ & 0.96 & 0.97 \\
$100 \mathrm{bar} / 77 \mathrm{~K} \rightarrow 5 \mathrm{bar} / 77 \mathrm{~K}$ & 0.96 & 0.99 \\
$100 \mathrm{bar} / 77 \mathrm{~K} \rightarrow 5 \mathrm{bar} / 160 \mathrm{~K}$ & 0.99 & 0.98 \\
\hline
\end{tabular}

*: Feynman-Hibbs correction

\section{Evaluating the performance of COFs for $\mathrm{H}_{2}$ storage:}

We identified the top performing COFs for $\mathrm{H}_{2}$ storage and ranked them based on their WCs computed from single-component GCMC simulations at 100 bar/77 K adsorption and 5 bar/77 K/ $160 \mathrm{~K}$ desorption conditions. Table 4 shows the top performing 10 COFs which exhibit the highest volumetric $\mathrm{H}_{2}$ WCs $(38.9-42.9 \mathrm{~g} / \mathrm{L})$ at $100 \mathrm{bar} / 77$ $\mathrm{K}$ adsorption and 5 bar/77 $\mathrm{K}$ desorption conditions. The WCs here were obtained from the results of GCMC simulations with the Feynman-
Hibbs correction using the Darkrim and Levesque potential for $\mathrm{H}_{2}$ (only $\mathrm{H}_{2}-\mathrm{H}_{2}$ electrostatic interactions are on).

The densities (porosities) of the top 10 COFs range from $0.16 \mathrm{~g} / \mathrm{cm}^{3}$ to $0.24 \mathrm{~g} / \mathrm{cm}^{3}$ (0.870.92 ). The top materials have all $3 D$ structures except IISERP-COF3 which has 2D structure. COF-DL-229-3-fold has the highest $\mathrm{H}_{2}$ WC (42.9 $\mathrm{g} / \mathrm{L})$ among 296 COFs which can be attributed to its high porosity (0.87) and large surface area (8462.g $\left.\mathrm{m}^{2} / \mathrm{g}\right)$.

Table 4: Top performing 10 COFs ranked based on their $\mathrm{H}_{2}$ WCs calculated at 100 bar/77 K adsorption and 5 bar/77 $\mathrm{K}$ desorption conditions.

\begin{tabular}{lllll}
\hline COF & LCD-PLD $(\AA)$ & $\mathbf{p}\left(\mathbf{g} / \mathbf{c m}^{\mathbf{3}}\right)$ & $\boldsymbol{\varphi}$ & $\mathbf{W C}(\mathbf{g} / \mathbf{L})$ \\
\hline COF-DL[1]229-3-fold & $10.72-10.15$ & 0.24 & 0.87 & 42.92 \\
PI $^{[2]-C O F-5-2 P[3] ~}$ & $13.35-10.52$ & 0.26 & 0.88 & 41.14 \\
COF-DL229-2-fold & $17.57-14.36$ & 0.16 & 0.92 & 40.78 \\
DL-COF-1-ctn & $16.21-14.26$ & 0.19 & 0.90 & 40.67 \\
DL-COF-2-ctn & $16.19-14.24$ & 0.21 & 0.90 & 40.47 \\
COF-105 & $18.80-16.12$ & 0.18 & 0.91 & 39.63 \\
DL-COF-1-bor & $22.72-16.03$ & 0.17 & 0.91 & 39.41 \\
IISERP[4]-COF3 & $22.16-19.77$ & 0.22 & 0.90 & 39.15 \\
Ni-DBA[5]-3D-COF-ctn & $20.96-17.60$ & 0.17 & 0.92 & 39.04 \\
DL-COF-2-bor & $25.15-15.94$ & 0.19 & 0.91 & 38.87
\end{tabular}

[1] DL: dual linkage; [2] PI: polyimide; [3] 2P: biphenyl-4,4'-dicarboxaldehyde; [4] IISERP:Indian Institute of Science Education and Research Pune; [5] DBA: dehydrobenzoannulenes. 
It is also important to note that COF-DL-229-3fold has smaller pore sizes $(10.72 \times 10.15 \AA)$ compared to the remaining 9 COFs which enhance $\mathrm{H}_{2}-\mathrm{COF}$ interactions. Bucior et al. (1) also discussed that large-pored materials exhibit weak $\mathrm{H}_{2}$-adsorbent interactions to bind $\mathrm{H}_{2}$ molecules.

We also ranked 296 COFs based on their volumetric $\mathrm{H}_{2}$ WCs calculated at 100 bar/77 K adsorption and 5 bar $/ 160 \mathrm{~K}$ desorption conditions and listed in Table 5 . Since adsorption is an exothermic process, when the desorption temperature increased from $77 \mathrm{~K}$ to $160 \mathrm{~K}, \mathrm{H}_{2}$ uptakes in COFs decreased as expected. Since $\mathrm{H}_{2}$ WCs were calculated using the difference between $\mathrm{H}_{2}$ uptake amounts at adsorption and des- orption pressures, volumetric $\mathrm{H}_{2}$ WCs (52.7-58.0 $\mathrm{g} / \mathrm{L})$ were increased as shown in Table 5 . COF103 exhibited the highest volumetric $\mathrm{H}_{2}$ WC as $58.0 \mathrm{~g} / \mathrm{L}$ at 100 bar/77K adsorption and 5 bar/ $160 \mathrm{~K}$ desorption conditions. The top performing materials listed in Table 5 have higher densities $\left(0.24-0.47 \mathrm{~g} / \mathrm{cm}^{3}\right)$ compared to the top candidates listed in Table 4 whereas they have narrower pore sizes and lower porosities than the latter. These materials also have 3D structures except ILCOF-1-AB. Among these 20 materials, COF-DL229-3fold is the common COF that exhibits high performance for $\mathrm{H}_{2}$ capture at two different adsorption/desorption conditions. Results showed that different operating conditions should be considered in order to identify the best performing COFs for $\mathrm{H}_{2}$ storage.

Table 5: Top performing 10 COFs ranked based on their $\mathrm{H}_{2}$ WCs calculated at 100 bar/77K adsorption and 5 bar/160K desorption conditions

\begin{tabular}{lllll}
\hline COF & LCD-PLD $(\AA)$ & $\mathbf{p}\left(\mathbf{g} / \mathbf{c m}^{\mathbf{3}}\right)$ & $\boldsymbol{\varphi}$ & $\mathbf{W C}(\mathbf{g} / \mathbf{L})$ \\
\hline COF-103 & $9.68-8.50$ & 0.39 & 0.80 & 58.04 \\
ILCOF$^{[1]}-1-A B$ & $11.09-9.41$ & 0.34 & 0.82 & 56.71 \\
COF-102 & $9.04-7.99$ & 0.42 & 0.78 & 56.52 \\
3D-Py[2]-COF-2P & $13.47-12.29$ & 0.28 & 0.85 & 56.06 \\
BF[3]-COF-1 $_{\text {COF-DL229-3fold }}^{13.26-8.62}$ & 0.40 & 0.79 & 54.63 \\
BF-COF-2 & $10.72-10.15$ & 0.24 & 0.87 & 54.13 \\
3D-CuPor-COF & $13.28-7.58$ & 0.47 & 0.78 & 53.87 \\
3D-Por[4]-COF & $16.46-13.64$ & 0.33 & 0.84 & 53.47 \\
COF-DL229-5fold & $16.31-13.66$ & 0.31 & 0.84 & 53.26 \\
\hline
\end{tabular}

[1] ILCOF: imine-linked; [2] Py: pyrene-based; [3] BF: base-functionalized; [4] Por: porphyrin.

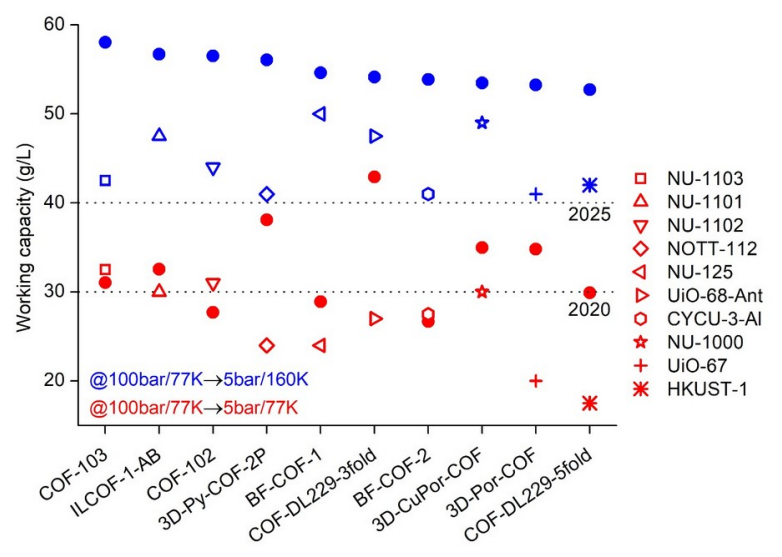

Figure 4: Comparison of volumetric $\mathrm{H}_{2}$ WCs of the top performing 10 COFs (closed spheres) with those of the promising MOFs (open symbols) identified in the literature. (9)

Figure 4 shows the comparison of volumetric $\mathrm{H}_{2}$ WCs of the top performing 10 COFs (listed in Table 5) studied under different temperature and pressure conditions with those of the best-performing MOFs at the same conditions. All porous materials surpassed the DOE target for 2025 at 100 bar/77 K adsorption and 5 bar/160K desorption conditions. Among 10 COFs, only ILCOF-1-
$A B$ has 2D structure, the remaining COFs are all 3D. As shown in Figure 4, COFs also outperformed the top performing 10 MOFs including HKUST-1 (also known as Cu-BTC, BTC: benzene1,3,5-tricarboxylate), NU-125, NU-1000, NU1101, NU-1102,

NU-1103, NOTT-112 (NOTT: University of Nottingham), UiO-68-Ant (UiO: University of Oslo; Ant: anthracene), UiO-67 and CYCU-3-Al (CYCU: Chung-Yuan Christian University; Al: aluminum) at 100 bar/77 K adsorption and 5 bar/160 K desorption conditions. Among 10 COFs, COFDL229-3-fold exhibited promising performance for $\mathrm{H}_{2}$ storage at two different operating conditions, exceeding the DOE 2020 and 2025 targets. This can be attributed to optimal pore sizes $(10.72 \AA \times 10.15 \AA)$ of COF-DL229-3-fold, its adequate porosity $(0.87)$ and density $(0.24 \mathrm{~g} /$ $\mathrm{cm}^{3}$ ). Bobbitt et al. (37) also discussed that the materials, which have optimal porosity $(\sim 0.9)$ and pore sizes $(\sim 12 \AA)$, tend to strongly bind with $\mathrm{H}_{2}$ molecules, resulting in high $\mathrm{H}_{2}$ uptake capacity.

As shown in Figure 4, NU-1103 (32.5 g/L) outperformed BF-COF-1, BF-COF-2, COF-102, COF103, and COF-DL229-5-fold at 100 bar/77 K ad- 
sorption and 5 bar/77 $\mathrm{K}$ desorption conditions. This might be explained that NU-1103 has lower density $\left(0.29 \mathrm{~g} / \mathrm{cm}^{3}\right)$ and higher porosity $(0.88)$ than these 5 COFs, whose densities were around $0.4 \mathrm{~g} / \mathrm{cm}^{3}$ and porosities $\sim 0.80$. Moreover, NU1000, NU-1101 and NU-1102 have slightly higher porosities $(\sim 0.80)$ than BF-COF-1, BFCOF-2, and COF-102 (0.78), and these MOFs $(\sim 30.5 \mathrm{~g} / \mathrm{L})$ also outperformed $3 \mathrm{COFs}$ at the same operating conditions. At $100 \mathrm{bar} / 77 \mathrm{~K}$ adsorption and 5 bar/77 $\mathrm{K}$ desorption conditions, BF-COF-2 gave almost similar $\mathrm{H}_{2}$ WC with CYCU3-Al due to their similar densities $(\sim 0.45 \mathrm{~g} /$ $\mathrm{cm}^{3}$ ). The other 4 COFs, namely as ILCOF-1-AB, 3D-Py-COF-2P, 3D-CuPor-COF, and 3D-Por-COF gave higher $\mathrm{H}_{2}$ WCs than MOFs at two operating conditions. Overall, COFs can exceed the DOE 2020 and 2025 targets for on board $\mathrm{H}_{2}$ storage.

Developing relationships between structural characteristics of materials and their performance for gas adsorption is important to better understand the behavior of materials and to synthesize promising candidates for desired applications. For this reason, we finally examined the structure-performance relationships for 296 COFs studied in this work. We investigated the relations between volumetric $\mathrm{H}_{2}$ WCs of COFs and their structural properties including the LCD, PLD, SA, density $(\rho)$, UV (Unit cell volume), PV, and porosity $(\varphi)$ in Figure 5.

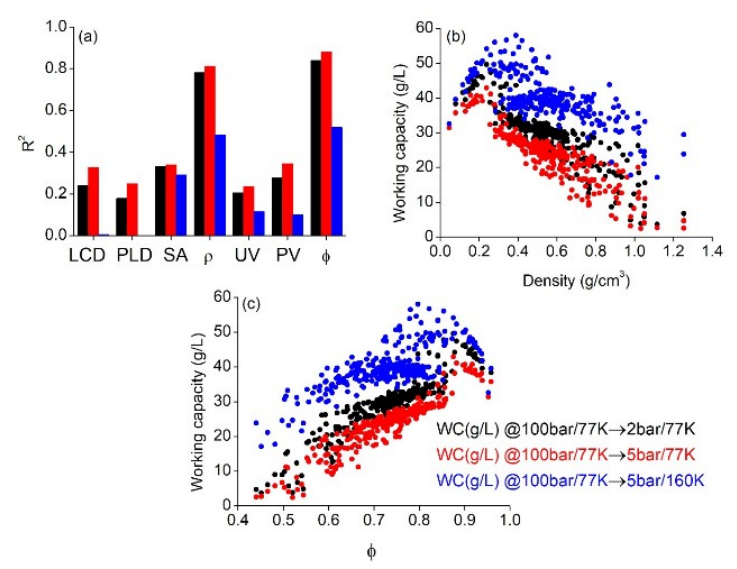

Figure 5: (a) $\mathrm{R}^{2}$ values showing the relations between WC of $\mathrm{H}_{2}(\mathrm{~g} / \mathrm{L})$ and several physical parameters. Relations between (b) density and $\mathrm{H}_{2}$

working capacity ( $\mathrm{g} / \mathrm{L})$, (c) porosity and $\mathrm{H}_{2}$ working capacity $(\mathrm{g} / \mathrm{L})$.

As shown in Figure 5 (a), correlation coefficients $\left(R^{2}\right)$ were found to be higher than 0.8 for the relations between volumetric WCs and $\rho$, and $\varphi$ of COFs for two operating conditions: at $100 \mathrm{bar} /$ $77 \mathrm{~K}$ for adsorption and at 2 bar/77 K (also 5 bar/77 K) for desorption. For the third case (from 100 bar/77 $\mathrm{K}$ to 5 bar/160 K), we did not observe a strong correlation $\left(R^{2}=0.5\right)$ between volumetric $\mathrm{H}_{2}$ WCs and $\rho$, and $\varphi$ of COFs. Weak correlations between volumetric $\mathrm{H}_{2}$ WCs of COFs and their LCDs, PLDs, SAs, UVs, PVs were observed for each three operational conditions as shown in Figure 5 (a).

Figure 5(b) shows the relationships between volumetric $\mathrm{H}_{2}$ WCs of COFs and their densities (ranged from 0.05 to $1.25 \mathrm{~g} / \mathrm{cm}^{3}$ ) at three operating conditions. $\mathrm{H}_{2}$ WCs of COFs are negatively correlated with the density, especially $\rho>0.3 \mathrm{~g} /$ $\mathrm{cm}^{3}$, at these conditions. The materials which have densities in the range of $0.12 \mathrm{~g} / \mathrm{cm}^{3}$ and $0.26 \mathrm{~g} / \mathrm{cm}^{3}$ exhibited generally high $\mathrm{H}_{2}$ WCs (32.4-54.1 g/L). For example, COF-DL229-3fold were found to have the highest $\mathrm{H}_{2}$ WCs as 49.62 $\mathrm{g} / \mathrm{L}(42.92 \mathrm{~g} / \mathrm{L}$ ) at $100 \mathrm{bar} / 77 \mathrm{~K} \rightarrow 2 \mathrm{bar} / 77 \mathrm{~K}$ (at $100 \mathrm{bar} / 77 \mathrm{~K} \rightarrow 5 \mathrm{bar} / 77 \mathrm{~K}$ ) due to its optimal density $\left(0.24 \mathrm{~g} / \mathrm{cm}^{3}\right)$ and high porosity (0.87). In Figure 5(c), the linear relationships between $\mathrm{H}_{2}$ WCs of COFs and their porosities (ranged from 0.44 to 0.96 ) can be seen. The materials which have high porosities $(\geq 0.8)$ and quite low densities $\left(0.2-0.4 \mathrm{~g} / \mathrm{cm}^{3}\right)$ generally exhibit high volumetric $\mathrm{H}_{2}$ WCs.

For example, COF-103, which gave the maximum $\mathrm{H}_{2}$ WC at 100 bar/77 $\mathrm{K} \rightarrow 5$ bar/160K, has high porosity $(0.80)$ and quite low density $(\sim 0.4$ $\left.\mathrm{g} / \mathrm{cm}^{3}\right)$. On the other hand, COF-DL229-0-fold has the highest porosity (0.96), however exhibited an average performance for $\mathrm{H}_{2}(\sim 35 \mathrm{~g} / \mathrm{L})$ at three operating conditions due to its extremely low density $\left(0.08 \mathrm{~g} / \mathrm{cm}^{3}\right)$ and very large pore sizes $(24.6 \times 31.9 \AA)$. Results emphasized that volumetric $\mathrm{H}_{2}$ storage in COFs can be negatively (positively) correlated with the densities (porosities) of COFs, like MOFs. It is also important to note that among 296 COFs, 257 COFs have 2D structures, and the remaining 39 COFs which commonly show high performance for $\mathrm{H}_{2}$ storage are 3D.

\section{CONCLUSIONS}

In this work, $\mathrm{H}_{2}$ storage performance of 296 COFs was assessed at various temperatures and pressures. Top 10 COFs, which gave the highest volumetric $\mathrm{H}_{2}$ WCs were identified in different operating conditions. Effects of electrostatic interactions and the Feynman-Hibbs correction on the ranking of the top materials were examined and results showed that coulombic interactions and the Feynman-Hibbs correction do not actually affect the ranking of COFs based on their $\mathrm{H}_{2}$ WCs. Among 296 COFs, COF-DL229-3fold outper-formed the ultimate DOE 2020 and DOE 2025 technical targets for on board $\mathrm{H}_{2}$ storage. The COFs which have high porosities $(\geq 0.8)$ and ade-quate densities $\left(0.2-0.4 \mathrm{~g} / \mathrm{cm}^{3}\right)$ exhibited a promising performance in terms of volumetric $\mathrm{H}_{2}$ 
storage. Results of this study will be helpful for future experimental and computational studies to design and synthesize novel COFs for $\mathrm{H}_{2}$ capture.

\section{REFERENCES}

1. Bucior BJ, Bobbitt NS, Islamoglu T, Goswami S, Gopalan A, Yildirim T, Farha OK, Bagheri N, Snurr RQ. Energy-based descriptors to rapidly predict hydrogen storage in metal-organic frameworks. Molecular Systems Design \& Engineering. 2019;4(1):162-74.

2. Gómez-Gualdrón DA, Wang TC, García-Holley P, Sawelewa RM, Argueta E, Snurr RQ, Hupp JT, Yildirim T, Farha OK. Understanding volumetric and gravimetric hydrogen adsorption trade-off in metal-organic frameworks. ACS Applied Materials \& Interfaces. 2017;9(39):33419-28.

3. Mason JA, Veenstra M, Long JR. Evaluating metal-organic frameworks for natural gas storage. Chemical Science. 2014;5(1):32-51.

4. Stadie NP, Vajo JJ, Cumberland RW, Wilson AA, Ahn CC, Fultz B. Zeolite-templated carbon materials for high-pressure hydrogen storage. Langmuir. 2012;28(26):10057-63.

5. Vitillo JG, Ricchiardi G, Spoto G, Zecchina A. Theoretical maximal storage of hydrogen in zeolitic frameworks. Physical Chemistry Chemical Physics. 2005;7(23):3948-54.

6. Farha OK, Yazaydın AÖ, Eryazici I, Malliakas CD, Hauser BG, Kanatzidis MG, Nguyen ST, Snurr RQ, Hupp JT. De novo synthesis of a metal-organic framework material featuring ultrahigh surface area and gas storage capacities. Nature Chemistry. 2010;2(11):944-8.

7. Han SS, Furukawa H, Yaghi OM, Goddard Iii WA. Covalent organic frameworks as exceptional hydrogen storage materials. Journal of the American Chemical Society. 2008;130(35):11580-1.

8. Rabbani MG, Sekizkardes AK, Kahveci Z, Reich TE, Ding R, El-Kaderi HM. A 2D mesoporous imine-linked covalent organic framework for high pressure gas storage applications. Chemistry-A European Journal. 2013;19(10):3324-8.

9. García-Holley $P$, Schweitzer $B$, Islamoglu $T$, Liu Y, Lin L, Rodriguez S, Weston MH, Hupp JT, Gómez-Gualdrón DA, Yildirim T. Benchmark study of hydrogen storage in metal-organic frameworks under temperature and pressure swing conditions. ACS Energy Letters. 2018;3(3):74854.
10. Suh MP, Park HJ, Prasad TK, Lim D-W. Hydrogen storage in metal-organic frameworks. Chemical Reviews. 2011;112(2):782-835.

11. Siegel D, Hardy B, Team H. Engineering an adsorbent-based hydrogen storage system: what have we learned 2015 [Available from: https://energy.gov/sites/prod/files/2015/02/f19/ fcto_h2_storage_summit_siegel.pdf

12. Langmi HW, Ren J, North B, Mathe M, Bessa-rabov D. Hydrogen storage in metalorganic frameworks: a review. Electrochimica Acta. 2014;128:368-92.

13. Gulcay E, Erucar I. Molecular simulations of COFs, IRMOFs and ZIFs for adsorption-based separation of carbon tetrachloride from air. Journal of Molecular Graphics and Modelling. 2019;86:84-94.

14. Furukawa $\mathrm{H}$, Yaghi OM. Storage of hydrogen, methane, and carbon dioxide in highly porous covalent organic frameworks for clean energy applications. Journal of the American Chemical Society. 2009;131(25):8875-83.

15. Ding S-Y, Wang W. Covalent organic frameworks (COFs): from design to applications. Chemical Society Reviews. 2013;42(2):548-68.

16. Basdogan $Y$, Keskin S. Simulation and modelling of MOFs for hydrogen storage. CrystEngComm. 2015;17(2):261-75.

17. Ahmed A, Seth S, Purewal J, Wong-Foy AG, Veenstra M, Matzger AJ, Siegel DJ. Exceptional hydrogen storage achieved by screening nearly half a million metal-organic frameworks. Nature Communications. 2019;10(1):1568.

18. Cao D, Lan J, Wang W, Smit B. Lithiumdoped 3D covalent organic frameworks: highcapacity hydrogen storage materials. Angewandte Chemie International Edition. 2009;48(26):4730-3.

19. Assfour B, Seifert G. Adsorption of hydrogen in covalent organic frameworks: comparison of simulations and experiments. Microporous and Mesoporous Materials. 2010;133(1-3):59-65.

20. Tong M, Lan Y, Qin Z, Zhong C. Computation-ready, experimental covalent organic framework for methane delivery: screening and material design. Journal of Physical Chemistry C. 2018;122(24):13009-16.

21. Li Z, Feng $X$, Zou $Y$, Zhang $Y$, Xia H, Liu X, $\mathrm{Mu} Y$. A 2D azine-linked covalent organic framework for gas storage applications. Chemical Communications. 2014;50(89):13825-8. 
22. Stegbauer L, Hahn MW, Jentys A, Savasci GK, Ochsenfeld C, Lercher JA, Lotsch BV. Tunable water and $\mathrm{CO} 2$ sorption properties in isostructural azine-based covalent organic frameworks through polarity engineering. Chemistry of Materials. $2015 ; 27(23): 7874-81$.

23. Li Z, Zhi $Y$, Feng $X$, Ding $X$, Zou $Y$, Liu X, Mu $Y$. An azine-linked covalent organic framework: synthesis, characterization and efficient gas storage. Chemistry-A European Journal. 2015;21(34):12079-84.

24. Ge R, Hao D, Shi Q, Dong B, Leng W, Wang C, Gao Y. Target synthesis of an azo $(\mathrm{N}=\mathrm{N})$ based covalent organic framework with high $\mathrm{CO} 2-$ over-N2 selectivity and benign gas storage capability. Journal of Chemical \& Engineering Data. 2016;61(5):1904-9.

25. Neti VSPK, Wu $X$, Hosseini M, Bernal RA, Deng $S$, Echegoyen L. Synthesis of $a$ phthalocya-nine 2D covalent organic framework. CrystEng-Comm. 2013;15(36):7157-60.

26. Kaleeswaran $D$, Vishnoi $P$, Murugavel $R$. $[3+3]$ Imine and $\beta$-ketoenamine tethered fluorescent covalent-organic frameworks for $\mathrm{CO} 2$ uptake and nitroaromatic sensing. Journal of Materials Chemistry C. $2015 ; 3(27): 7159-71$.

27. Kang $Z$, Peng $Y$, Qian $Y$, Yuan $D$, Addicoat MA, Heine T, Hu Z, Tee L, Guo Z, Zhao D. Mixed matrix membranes (MMMs) comprising exfoliated 2D covalent organic frameworks (COFs) for efficient $\mathrm{CO} 2$ separation. Chemistry of Materials. 2016;28(5):1277-85.

28. Bhunia A, Vasylyeva V, Janiak C. From a supramolecular tetranitrile to a porous covalent triazine-based framework with high gas uptake capacities. Chemical Communications. 2013;49(38):3961-3.

29. Kahveci Z, Islamoglu T, Shar GA, Ding R, ElKaderi HM. Targeted synthesis of a mesoporous triptycene-derived covalent organic framework. CrystEngComm. 2013;15(8):1524-7.

30. Willems TF, Rycroft $\mathrm{CH}$, Kazi M, Meza JC, Haranczyk M. Algorithms and tools for highthroughput geometry-based analysis of crystalline porous materials. Microporous and Mesoporous Materials. 2012;149(1):134-41.

31. Dubbeldam D, Calero S, Ellis DE, Snurr RQ. RASPA: molecular simulation software for adsorption and diffusion in flexible nanoporous materials. Molecular Simulation. 2016;42(2):81101.
32. Ewald PP. Die berechnung optischer und elektrostatischer gitterpotentiale. Annalen der Physik. 1921;369(3):253-87.

33. Buch V. Path integral simulations of mixed para-D2 and ortho-D2 clusters: The orientational effects. Journal of Chemical Physics. 1994;100(10): 7610-29.

34. Darkrim F, Levesque D. Monte Carlo simulations of hydrogen adsorption in single-walled carbon nanotubes. The Journal of Chemical Physics. $1998 ; 109(12): 4981-4$.

35. Feynman R, Hibbs A. Quantum mechanics and path integrals: McGraw-Hill, New York; 1965.

36. Rappé AK, Casewit CJ, Colwell K, Goddard III WA, Skiff WM. UFF, a full periodic table force field for molecular mechanics and molecular dynamics simulations. Journal of the American Chemical Society. 1992;114(25):10024-35.

37. Bobbitt NS, Chen J, Snurr RQ. Highthroughput screening of metal-organic frameworks for hydrogen storage at cryogenic temperature. Journal of Physical Chemistry C. 2016;120(48):27328-41. 
Gulcay E, Erucar İ. JOTCSA. 2020; 7(1): 65-76.

RESEARCH ARTICLE 